\title{
Barreiras para o acesso ao tratamento da catarata senil na Fundação Altino Ventura
}

\author{
Barriers in the access to senile cataract treatment at Altino Ventura Foundation
}

\author{
Danielle Maria Gomes de Lima ${ }^{1}$ \\ Liana Oliveira Ventura ${ }^{2}$ \\ Carlos Teixeira Brandt ${ }^{3}$
}

Este estudo foi realizado na Fundação Altino Ventura como pré-requisito à conclusão do Mestrado Profissionalizante/MBA da Prática Oftalmológica da Universidade Federal de São Paulo (UNIFESP).

${ }^{1}$ Oftalmologista do Hospital de Olhos de Pernambuco e Fundação Altino Ventura. Mestranda Profissional em Administração da Prática Oftalmológica da Universidade Federal de São Paulo (UNIFESP). São Paulo (SP). ${ }^{2}$ Oftalmologista Pediátrica do Hospital de Olhos de Pernambuco e Coordenadora da Fundação Altino Ventura. ${ }^{3}$ Professor titular de cirurgia pediátrica da Universidade Federal de Pernambuco. Professor Orientador do Mestrado Profissionalizante em Administração da Prática Oftalmológica da UNIFESP. São Paulo (SP).

Endereço para correspondência: Fundação Altino Ventura. Rua da Soledade, 170 - Recife (PE) CEP 50070-040

E-mail: fav@fundacaoaltinoventura.org.br leonardo.a.macedo@uol.com.br

Recebido para publicação em 04.08.2003 Versão revisada recebida em 07.12.2004 Aprovação em 03.03.2005

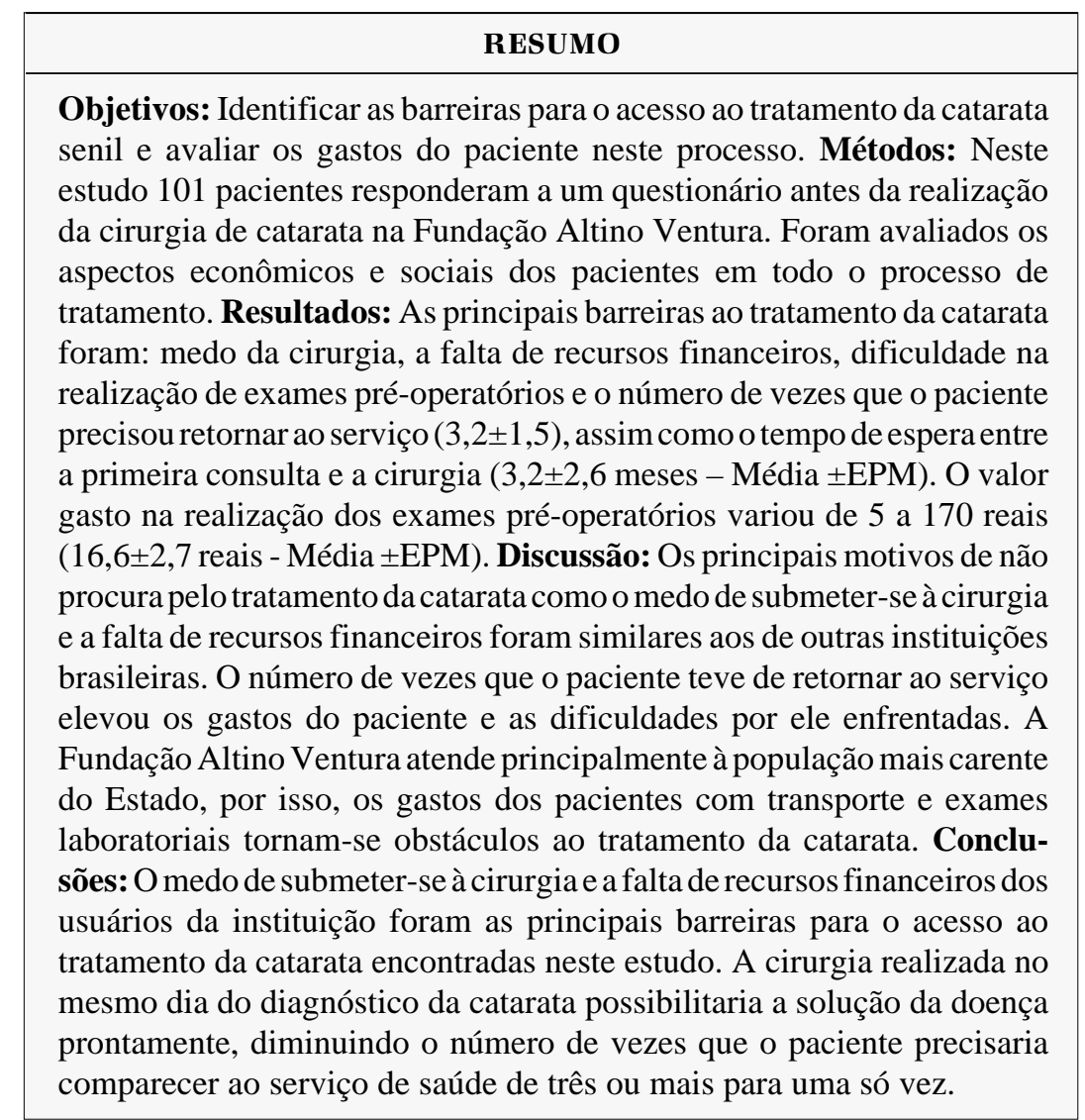

Descritores: Extração de catarata; Extração de catarata/economia; Cegueira/prevenção \& controle; Acesso aos serviços de saúde; Qualidade dos cuidados de saúde

\section{INTRODUÇ̃̃̃O}

A catarata é a causa de cegueira mais freqüente em todo o mundo, acometendo $75 \%$ dos indivíduos acima de 70 anos. Nos países em desenvolvimento, constitui-se em grave problema de saúde pública ${ }^{(1-2)}$.

A perda da capacidade visual afeta diretamente a qualidade de vida dos pacientes, dificultando a realização de atividades do cotidiano e a leitura. $\mathrm{O}$ déficit visual também dá origem a problemas psicológicos, sociais e econômicos, pois implica em perda da auto-estima, em restrições ocupacionais e conseqüente diminuição da renda familiar. Para a sociedade representa encargo oneroso e perda da força de trabalho ${ }^{(3-4)}$.

$\mathrm{O}$ aumento da expectativa de vida e da população idosa tem aumentado a incidência de catarata no mundo. O número de cirurgias de catarata 
realizadas por ano no Brasil, aumentou de 90 mil no início da década de 90 para cerca de 250 mil em 2.000. Entretanto, estima-se que seria necessária a realização de cerca de $450 \mathrm{mil}$ cirurgias por ano, para atender a demanda do Brasil ${ }^{(5)}$.

O tratamento cirúrgico dessa doença possibilita a restauração da visão e a reabilitação dos pacientes, possibilitando a sua reinserção à sociedade, inclusive com o retorno a algumas atividades laborais ${ }^{(6-9)}$.

Barreiras de ordem socioeconômicas e culturais dificultam o acesso dos portadores de catarata aos serviços de saúde, mesmo nas grandes capitais ${ }^{(9-15)}$.

A necessidade de exames pré-operatórios aumenta o número de vezes que o paciente precisa retornar ao hospital até a realização da cirurgia, funcionando como entrave no acesso ao tratamento da catarata. Todas essas barreiras devem ser analisadas no seu contexto local a fim de que se consiga diminuir ou mesmo prevenir a cegueira por catarata ${ }^{(15-34)}$.

Os objetivos deste estudo foram identificar as barreiras dos pacientes para o acesso ao tratamento da catarata senil na Fundação Altino Ventura e os custos do paciente no processo de tratamento.

\section{MÉTODOS}

Participaram deste estudo pacientes portadores de catarata senil, antes da realização da cirurgia de catarata (facectomia), na sede da Fundação Altino Ventura, entre setembro e novembro de 2002. Os pacientes responderam verbalmente a um questionário (Anexo 1) onde foram avaliados os dados quanto a gênero, idade, escolaridade, profissão, procedência, tempo de baixa visual e dificuldades em realizar tarefas do diaa-dia pela baixa visual.

Os pacientes responderam se tinham conhecimento prévio da sua doença (catarata) antes de ir à Fundação e o motivo da não procura pelo tratamento. $\mathrm{O}$ acesso ao serviço de saúde foi avaliado através do questionamento quanto à forma de conhecimento da Instituição, o tempo de espera para a realização da primeira consulta, o número de vezes que o paciente compareceu ao serviço até a cirurgia, o tempo entre a primeira consulta e a realização da cirurgia e as despesas do paciente com transporte e exames.

Os resultados foram expressos por suas freqüências. As variáveis contínuas foram expressas por suas médias e respectivos desvios-padrão, ou, o erro padrão da média (EPM), quando o grau de dispersão da variável era muito alto.

Os pacientes concordaram em participar do estudo assinando um termo de consentimento livre e esclarecido. O questionário aplicado foi previamente submetido à análise e aprovado pela Comissão de Ética em Pesquisa da Fundação Altino Ventura e Universidade Federal de São Paulo.

\section{RESULTADOS}

A amostra compôs-se de 101 pacientes: 70 (69,3\%) mulheres e $31(30,7 \%)$ homens. A idade variou de 41 a 94 anos, com

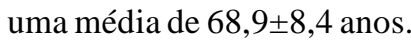

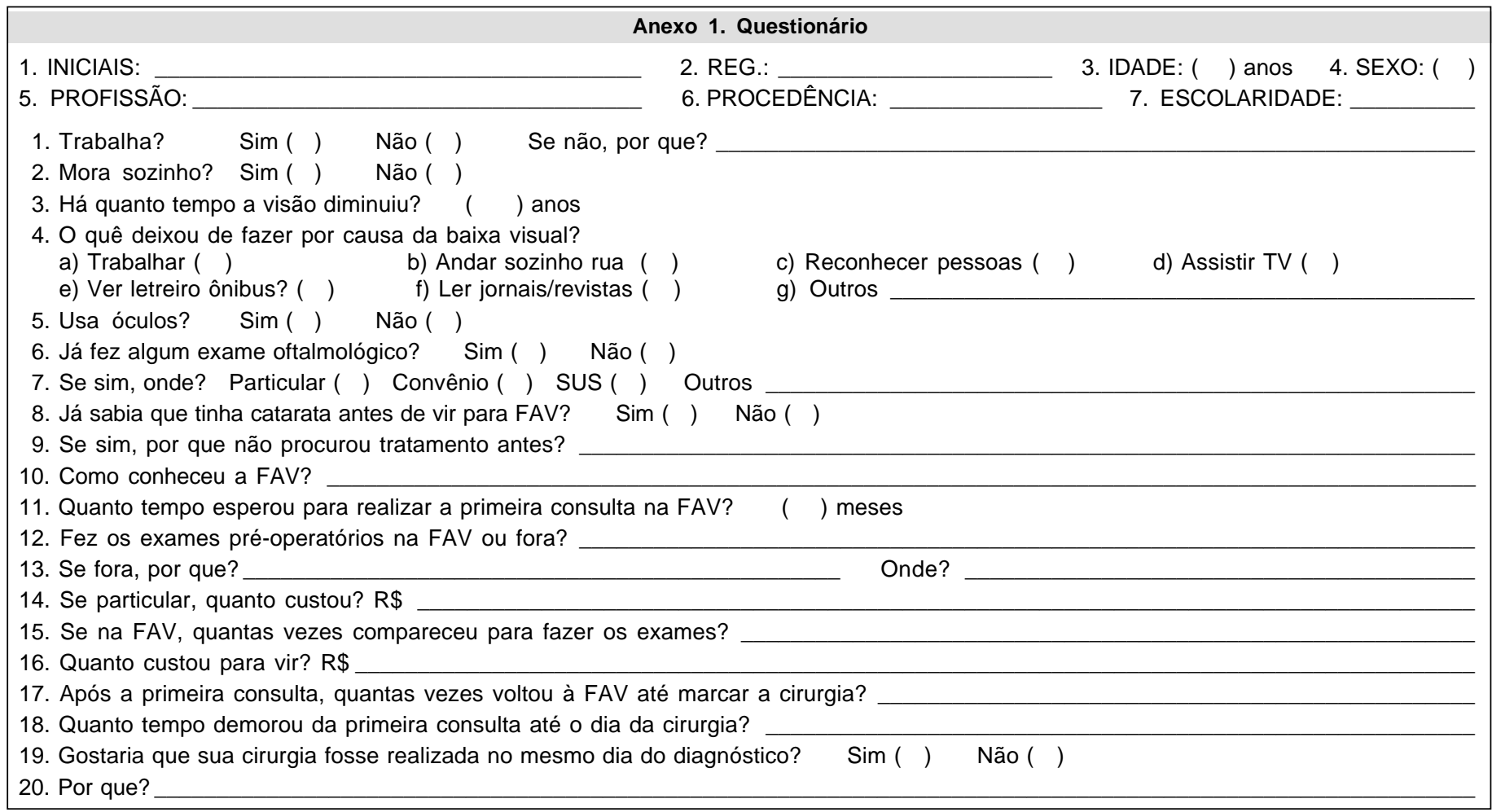


Os pacientes eram, na sua maioria, aposentados (Gráfico 1). Quanto à procedência, 40 pacientes $(39,6 \%)$ eram de Recife, $55(54,5 \%)$ da Região Metropolitana e $6(5,9 \%)$ de cidades do interior do Estado.

A maioria dos pacientes tinha o primeiro grau incompleto (Gráfico 2).

As distribuições das freqüências dos motivos de não procura pelo tratamento encontram-se descritos na tabela 1 .

O tempo médio de espera pelo primeiro atendimento oftalmológico foi de $1,1 \pm 0,1$ meses - média \pm EPM). A distribuição das frequiências da forma de conhecimento da FAV está na tabela 2 .

A distribuição das freqüências dos pacientes quanto à capacidade de desenvolver atividades do cotidiano está na tabela 3 .

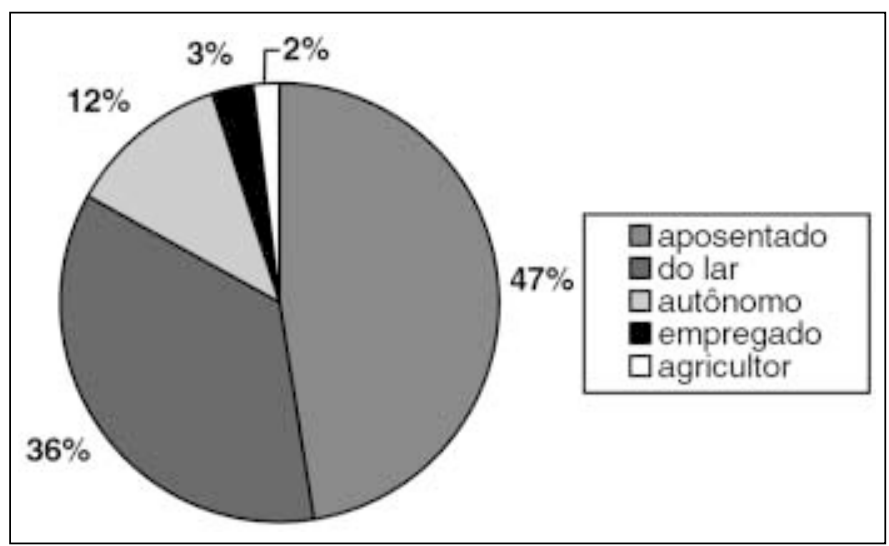

Gráfico 1 - Ocupação dos pacientes portadores de catarata. Fundação Altino Ventura. 2002

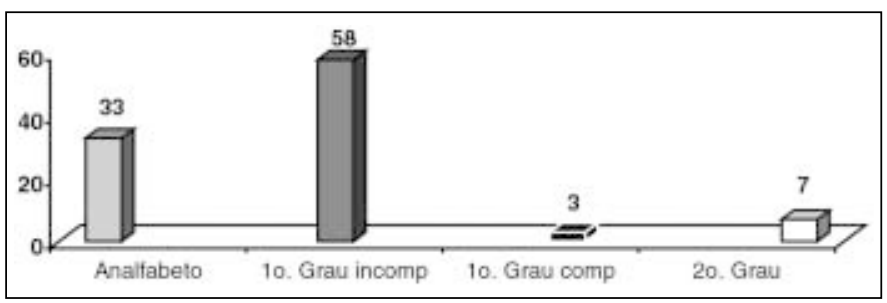

Gráfico 2 - Nível de escolaridade dos pacientes portadores de catarata.

Fundação Altino Ventura. 2002
Quanto ao tempo de baixa visual (BAV), 47 pacientes $(46,5 \%)$ referiram menos de dois anos de baixa visual, $40(39,6 \%)$ de dois a cinco anos e $14(13,9 \%)$ mais de cinco anos.

Dos pacientes que sabiam ler, $41(55,4 \%)$ não conseguiam mais ler pela BAV e dos que costuravam, 46 (79,3\%) não o faziam mais pela BAV.

Fizeram o exame oftalmológico pela primeira vez na FAV 12 $(11,9 \%)$ pacientes e $28(27,7 \%)$ não usavam óculos. Dos pacientes estudados, $89(88,1 \%)$ já tinham feito exame oftalmológico antes. Destes, $45(50,6 \%)$ pelo SUS, 34 (38,2\%) em clínicas particulares e $10(11,2 \%)$ por meio de planos de saúde.

Quanto aos conhecimentos prévios da doença, 49 (48,5\%) pacientes sabiam que tinham catarata antes do exame na FAV e destes, $21(42,9 \%)$ procuraram imediatamente a Instituição para a realização da cirurgia.

O número de vezes que o paciente compareceu a FAV até a data da cirurgia variou de uma a oito vezes, com uma média de $3,2 \pm 1,5$. O tempo médio desde a primeira consulta até a realização da cirurgia variou de um a 12 meses, com uma média de 3,2 $\pm 2,6$ meses (Média \pm EPM).

\begin{tabular}{|lcr|}
\hline \multicolumn{3}{|c|}{$\begin{array}{c}\text { Tabela 1. Motivos de não procura pelo tratamento da catarata. } \\
\text { Fundação Altino Ventura. }\end{array}$} \\
Motivo & $\mathbf{n}$ & $\%$ \\
Medo & 7 & 14,3 \\
Catarata não estava "madura" & 6 & 12,2 \\
Difícil acesso ao serviço de saúde & 5 & 10,2 \\
Descuido & 5 & 10,2 \\
Falta de tempo & 3 & 6,1 \\
Falta de recursos financeiros & 2 & 4,1 \\
Procurou logo o serviço da saúde & 21 & 42,9 \\
Total & 49 & 100,0 \\
\hline
\end{tabular}

Tabela 2. Forma de conhecimento da Instituição. Fundação Altino Ventura. 2002

Forma de conhecimento da FAV

Parentes, vizinhos ou amigos $\%$

Encaminhamento médico (oftalmologista ou não)

Encaminhamento médico (oftalmologista ou não) $26 \quad 25,8$

$\begin{array}{lll}\text { Mutirão de catarata no seu bairro ou cidade } & 16 & 15,8\end{array}$

Unidade móvel de atendimento clínico da FAV $\quad 11 \quad 10,9$

Total

$101 \quad 100,0$

\begin{tabular}{|c|c|c|c|c|c|c|}
\hline \multirow[t]{2}{*}{ Atividade* } & \multicolumn{2}{|c|}{ Sim } & \multicolumn{2}{|c|}{ Não } & \multicolumn{2}{|c|}{ Total } \\
\hline & $\mathbf{n}$ & $\%$ & $\mathbf{n}$ & $\%$ & $\mathbf{n}$ & $\%$ \\
\hline Trabalhar & 12 & 11,9 & 89 & 88,1 & 101 & 100 \\
\hline Morar sozinho & 18 & 17,8 & 83 & 82,2 & 101 & 100 \\
\hline Andar sozinho & 76 & 75,2 & 25 & 24,8 & 101 & 100 \\
\hline Reconhecer pessoas & 67 & 66,3 & 34 & 33,7 & 101 & 100 \\
\hline Assistir TV & 94 & 93,1 & 7 & 6,9 & 101 & 100 \\
\hline Ver letreiro do ônibus & 39 & 38,6 & 62 & 61,4 & 101 & 100 \\
\hline *Respostas múltiplas & & & & & & \\
\hline
\end{tabular}


Os exames pré-operatórios foram realizados fora da Instituição por 73 pacientes $(72,2 \%)$. Destes, $38(52,1 \%)$ alegaram demora em realizar os exames na FAV, $31(42,5 \%)$ a possibilidade de fazer os exames mais próximos à sua residência e $4(5,5 \%)$ a realização destes exames por planos de saúde. Os exames foram feitos em clínicas particulares por 40 pacientes $(54,8 \%)$, pelo SUS por $29(39,7 \%)$ e por plano de saúde por 4 pacientes $(5,5 \%)$.

$\mathrm{O}$ valor gasto na realização dos exames pré-operatórios variou de cinco a 170 reais, com um valor médio de $16,6 \pm 2,7$ reais (Média \pm EPM) (Tabela 4).

Os pacientes tiveram um gasto médio com transporte para a FAV de 7,6 $\pm 1,7$ reais (Média \pm EPM) (Tabela 5).

Entre os pacientes entrevistados, $78(77,2 \%)$ concordariam com a realização da cirurgia no mesmo dia do primeiro exame, quando a catarata foi diagnosticada. Dos pacientes que não concordariam, $15(65,2 \%)$ alegaram medo de operar no mesmo dia e $8(34,8 \%)$ a necessidade de realizar exames pré-operatórios.

\section{DISCUSSÃO}

A exemplo de outros estudos ${ }^{(3-9)}$, a maioria dos pacientes que compôs a amostra tinha baixa escolaridade e era economicamente inativa (aposentados ou donas de casa) e 88\% não realizavam nenhum tipo de trabalho.

Os principais motivos de não procura pelo tratamento da catarata como o medo de submeter-se à cirurgia e a falta de recursos financeiros foram similares aos de outras Instituições brasileiras ${ }^{(9-12)}$. Esses fatores apontam a falta de conhecimento dos pacientes a respeito da catarata e suas condições socioeconômicas como principais obstáculos à procura pelo seu tratamento.

Aproximadamente metade dos pacientes do estudo conheceu a Instituição por meio de parentes, vizinhos ou amigos, destacando a importância da propaganda verbal ("paciente

\begin{tabular}{|c|c|c|}
\hline Valor gasto nos exames pré-operatórios & $\mathbf{n}$ & $\%$ \\
\hline Nenhum & 58 & 57,4 \\
\hline Até $R \$ 20,00$ & 15 & 14,8 \\
\hline De $R \$ 21,00$ a $R \$ 50,00$ & 19 & 18,9 \\
\hline Mais de $R \$ 50,00$ & 9 & 8,9 \\
\hline Total & 101 & 100,0 \\
\hline
\end{tabular}

\begin{tabular}{|c|c|c|}
\hline & $n$ & $\%$ \\
\hline Nenhum & 53 & 525 \\
\hline Até $R \$ 10,00$ & 32 & 31,7 \\
\hline De $R \$ 11,00$ a $R \$ 50,00$ & 12 & 11,9 \\
\hline Mais de $R \$ 50,00$ & 4 & 3,9 \\
\hline Total & 101 & 100,0 \\
\hline
\end{tabular}

para paciente") como forma de divulgação dos serviços de saúde prestados por esta Fundação.

Destaca-se, ainda, a atuação da unidade móvel de atendimento clínico e dos mutirões de catarata desenvolvidos pela FAV, como captadores de pacientes, fazendo o diagnóstico na própria comunidade e os encaminhado ao tratamento adequado, diminuindo assim, a dificuldade de acesso ao serviço de saúde.

Não foi observada, neste estudo, dificuldade para a marcação da consulta na FAV, nem para marcação da cirurgia. Isto se deve a políticas da própria Instituição, que priorizam o atendimento a pacientes idosos, visando combater a cegueira por catarata, uma das metas desta organização.

A maioria dos indivíduos deste estudo já tinha realizado consulta oftalmológica anteriormente, entretanto, apenas a metade deles o fez pelo Sistema Único de Saúde e aproximadamente $40 \%$ por meio de clínicas particulares.

Observa-se uma dificuldade de acesso ao atendimento especializado em oftalmologia na rede pública. Isto se deve, provavelmente, ao pequeno número de clínicas especializadas no sistema público, o que gera importante demanda reprimida, que recorre, assim, ao setor privado.

Acredita-se que o crescente aumento das "clínicas populares", próximas à residência do paciente, tem possibilitado o acesso a esse tipo de serviço.

A necessidade de exames pré-operatórios foi importante barreira ao tratamento. Cerca de $70 \%$ desses exames foram feitos fora da Instituição. Os exames exigidos pelo Sistema Único de Saúde são: hemograma completo, coagulograma, glicemia de jejum e parecer cardiológico, entretanto, vários estudos apontam que os exames pré-operatórios para cirurgias de pequeno porte, incluindo cirurgias oftalmológicas, são desnecessários ${ }^{(16-34)}$. A solicitação destes exames contribui para uma menor eficiência dos serviços médicos disponibilizados para indivíduos de baixa condição socioeconômica.

O número de vezes que o paciente teve de retornar ao serviço elevou os gastos do paciente e as dificuldades por ele enfrentadas. A Fundação Altino Ventura atende principalmente à população mais carente do Estado, por isso, os gastos dos pacientes com transportes e exames laboratoriais tornamse obstáculos ao tratamento da catarata.

No presente estudo aproximadamente $80 \%$ dos pacientes concordaria com a realização da cirurgia no momento do diagnóstico. A maioria afirmou que confiava nos médicos e que gostaria da resolução rápida do problema para o restabelecimento da visão o mais depressa possível.

Com o advento da cirurgia ambulatorial (day surgery) os pacientes submetidos a cirurgias de pequeno e médio porte, têm alta hospitalar no mesmo dia da cirurgia, diminuindo os custos operacionais desses procedimentos ${ }^{(35-39)}$.

A cirurgia realizada no mesmo dia do diagnóstico da catarata possibilita a solução da doença prontamente, diminuindo o número de vezes que o paciente precisa comparecer ao serviço de saúde de três ou mais para uma só vez. O que permite um maior acesso da população ao tratamento cirúrgico da doença. 


\section{CONCLUSÕES}

As barreiras ao tratamento da catarata senil identificadas neste estudo foram: falta de conhecimento da doença, medo da cirurgia, dificuldade de realização dos exames pré-operatórios, número de retornos necessários até a data da cirurgia e despesas do paciente com os retornos ao serviço.

Há, portanto, uma lacuna entre a busca de serviços oftalmológicos e a resolução cirúrgica da catarata. É necessário facilitar o acesso da população à cirurgia de catarata por meio de modelos assistenciais descentralizados e campanhas comunitárias de prevenção da cegueira, onde o paciente possa ter o seu tratamento prontamente, com mínimo gasto e máxima segurança e eficiência.

\section{ABSTR ACT}

Purpose: To identify the barriers in the access to treatment of senile cataract and to evaluate the patient's costs in this process. Methods: A survey of 101 patients who would undergo cataract surgery at Altino Ventura Foundation was performed using a questionnaire. The economical and social aspects of the whole process of treatment were evaluated. Results: The principal barriers of cataract treatment were: fear of surgery, patient's low income, the need for preoperative examinations and the number of times the patient had to return to the service (3.2 \pm 1.5$)$, as well as the waiting time between the first examination and the surgery $(3.2 \pm 2.6$ months - Mean \pm SEM). The examination costs varied from 5 to 170 reais (16.6 \pm 2.7 - Mean \pm SEM). Discussion: The main reasons for not seeking for cataract treatment, as the fear to undergo surgery and the lack of financial resources had been similar to other Brazilian institutions. The number of times that the patient had to return to the service increased the expenses of the patient and the difficulties faced by him. Altino Ventura Foundation mainly takes care of the poorest population of the State, therefore, the expenses of the patients with transportation and examinations become obstacles to the treatment of cataract. Conclusions: The fear of surgery and the patient's low income and the social level of the institution's users were the principal barriers in the access to cataract treatment in this study. The need for preoperative examinations, sometimes unnecessary, contributes to a lower efficiency of the medical services offered to individuals of low income and social conditions. The surgery carried out at the same day of the cataract diagnosis makes the solution of the illness quickly possible, diminishing the number of times necessary for the patient's return to health service from three or more, to only one.

Keywords: Cataract extraction; Cataract extraction/economics; Blindness/prevention \& control; Health services accessibility; Quality of health care

\section{REFERÊNCIAS}

1. Monestan E, Wachtmeister L. The impact of cataract surgery on low vision patients: a population based study. Acta Ophthalmol Scand. 1997;75(5):569-76.

2. Resnikoff S, Pascolini D, Etyaále D, Kocur I, Pararajasegaram R, Pokharel GP, et al. Global data on visual impairment in the year 2000. Bull World Health Organ. 2002;82(11):844-51.

3. Ferraz EVA, Lima CA, Cella W, Arieta CEL. Adaptação de questionário de avaliação da qualidade de vida para aplicação em portadores de catarata. Arq Bras Oftalmol. 2002;65(3):293-8.

4. Pokharel GP, Selvaraj S, Ellwein LB. Visual functioning and quality of life outcomes among cataract operated and unoperated blind populations in Nepal. Br J Ophthalmol. 1998;82(6):606-10. Comment in: Br J Ophthalmol. 1998; 82(6):593-4.

5. País realiza 120 mil cirurgias extras de catarata. J Jota Zero nov/dez. 1999. p.23.

6. Rezende Filho F, Rezende F. Histórico. In: Rezende F. Cirurgia de catarata. Rio de Janeiro: Cultura Médica; 2001. p.3-8.

7. Leite Arieta CE, José NK, Carvalho Filho DM, Ruiz Alves M. Optimization of a university cataract-patient care service in Campinas, Brazil. Ophthalmic Epidemiol. 1999;6(2):113-23.

8. Kara-José Jr. N, Schellini SA, Silva MRB, Bruni LF, Almeida AGC. Projeto Catarata: qual a sua importância para a comunidade? Arq Bras Oftalmol. 1996;59(5):490-3.

9. Malerbi FK, Lopes JF, Ajimura FY, Alves MR, Temporini ER. Barreiras ao acesso no tratamento da catarata senil: aspectos sócio-culturais. Rev Bras Oftalmol. 2000;59(9):649-54.

10. Rehder JR, Ribeiro LGI, Melo Filho PAA, Aoki RH. "Projeto Catarata": uma solução para saúde pública ocular. Rev Bras Oftalmol. 1990;58(4):271-5.

11. Temporini ER, Kara-José N, Kara-José Jr N. Catarata senil: características e percepções de pacientes atendidos em projeto comunitário de reabilitação visual. Arq Bras Oftalmol. 1997;60(1):79-83.

12. Kara-José N, Arieta CEL, Temporini ER, Kang KM, Ambrosio LE. Tratamento cirúrgico de catarata senil: óbices para o paciente. Arq Bras Oftalmol. 1996; 59(6):573-7.

13. Kara-José N, Temporini ER. Cirurgia de catarata: o porquê dos excluídos. Rev Panam Salud Pública. 1999;6(4):242-8.

14. Scott IU, Schein OD, West S, Bandeen-Roche K, Enger C, Folstein MF. Functional status and quality of life measurement among ophthalmic patients. Arch Ophthalmol. 1994;112(3):329-35.

15. Prado Jr J, Silva ALB, Alves MR, Kara-José N, Temporini ER. Tratamento cirúrgico da catarata senil no Hospital das Clínicas da Faculdade de Medicina da Universidade de São Paulo. Rev Med (São Paulo). 1997;76(3):192-6.

16. Blery C, Chastang C, Gaudy JH. Critical assessment of routine preoperative investigations. Eff Health Care. 1983(2):111-4.

17. Kaplan EB, Sheiner LB, Boeckmann AJ, Roizen MF, Beal SL, Coho SN, et al. The usefulness of preoperative laboratory screening. JAMA. 1985;253(24):3576-81.

18. Fowkes FG. The value of routine preoperaative chest X-rays. Br J Hosp Med. 1986;35(2):120-3.

19. Blery C, Charpak Y, Ben Kemmoun R, Pham J, Brage D, Zindel G, et al. [Evaluation of a protocol for selective ordering of preoperative tests in healthy subjects]. Ann Fr Anesth Reanim. 1987;6(1):64-70. French.

20. McKee RF, Scott EM. The value of routine preoperative investigations. Ann R Coll Surg Engl. 1987;69(4):160-2.

21. Brandt CT. Avaliação laboratorial pré-operatória em cirurgia ambulatorial pediátrica. Arq Bras Med. 1988;62(4):261-4.

22. Johnson H Jr, Knee-Ioli S, Butler TA, Munoz E, Wise L. Are routine preoperative laboratory screening tests necessary to evaluate ambulatory surgical patients? Surgery. 1988;104(4):639-45.

23. McCleane GJ. Urea and electrolyte measurements in pre-operative surgical patients. Anaesthesia. 1988;43(5):413-5.

24. Macpherson DS, Snow R, Lofgren RP. Preoperative screening value of previous tests. Ann Intern Med. 1990;113(12):969-73.

25. Baron MJ, Gunter J, White P. Is the pediatric preoperative hematocrit determination necessary? South Med J. 1992;85(12):1187-9.

26. Walters G, McKibbin M. The value of pre-operative investigation in local anaesthetic ophthalmic surgery. Eye. 1997;11(Pt 6):847-9.

27. Ansermino JM, Than M, Swallow PD. Pre-operative blood tests in children undergoing plastic surgery. Ann R Coll Surg Engl. 1999;81(3):175-8.

28. Haug RH, Reifeis RL. A prospective evaluation of the value of preoperative laboratory testing for office anesthesia and sedation. J Oral Maxillofac Surg. 1999;57(1):21-2

29. Hesse S, Seebauer A, Schwender D. [Ambulatory anesthesia: which preoperative screening tests are required] Anaesthesist. 1999;48(2):108-15. German. 
30. Dzankic S, Pastor D, Gonzalez C, Leung JM. The prevalence and predictive value of abnormal preoperative laboratory tests in elderly surgical patients. Anesth Analg. 2001;93(2):301-8.

31. Szmuk P, Gurevich B, Dotan Z, Zabeeda D, Geva D, Ezri T. [The significance and cost of preoperative laboratory screening in young healthy patients in a public hospital in Israel]. Harefuah. 2002;14(4):344-6. Hebrew.

32. Alsumait BM, Alhumood SA, Ivanova T, Mores M, Edeia M. A prospective evaluation of preoperative screening laboratory tests in general surgery patients. Med Princ Pract. 2002;11(1):42-5.

33. Greer AE, Irwin MG. Implementation and evaluation of guidelines for preoperative testing in a tertiary hospital. Anaesth Intensive Care. 2002;30(3):326-30.

34. Johnson RK, Mortimer AJ. Routine pre-operative blood testing: is it necessary? Anesthesia. 2002;57(9):914-7.
35. Rose K, Waterman H, Toon L, McLeod D, Tullo A. Management of daysurgery patients with cataract attending a peripheral ophthalmic clinic. Eye. 1999;13(Pt 1):71-5.

36. Hughes EH, Forrest F, Diamond JP. "One stop" cataract surgery: the Bristol Eye Hospital experience 1997-1999. Eye. 2001;15(Pt 3):306-8.

37. Stephenson P. In a day's work. The government is opening 10 more diagnosis and treatment centers to reduce length of hospital stay, largely through day surgery. Health Serv J. 2002;112(5820):9-10.

38. Wertheim M, Burton R. Immediately sequential phacoemulsification performed under topical anaesthesia as day care procedures. Br J Ophthalmol. 2002; 86(12):1356-8.

39. Murthy GV, Sharma P. Cost analysis of eye camps and camp-based cataract surgery. Natl Med J India. 1994;7(3):111-4. 